TRANSACTIONS OF THE

AMERICAN MATHEMATICAL SOCIETY

Volume 361, Number 7, July 2009, Pages 3829-3850

S 0002-9947(09)04615-7

Article electronically published on March 4, 2009

\title{
WEIGHTED SOBOLEV SPACES AND EMBEDDING THEOREMS
}

\author{
V. GOL'DSHTEIN AND A. UKHLOV
}

\begin{abstract}
In the present paper we study embedding operators for weighted Sobolev spaces whose weights satisfy the well-known Muckenhoupt $A_{p^{-}}$ condition. Sufficient conditions for boundedness and compactness of the embedding operators are obtained for smooth domains and domains with boundary singularities. The proposed method is based on the concept of 'generalized' quasiconformal homeomorphisms (homeomorphisms with bounded mean distortion). The choice of the homeomorphism type depends on the choice of the corresponding weighted Sobolev space. Such classes of homeomorphisms induce bounded composition operators for weighted Sobolev spaces. With the help of these homeomorphism classes the embedding problem for nonsmooth domains is reduced to the corresponding classical embedding problem for smooth domains. Examples of domains with anisotropic Hölder singularities demonstrate the sharpness of our machinery comparatively with known results.
\end{abstract}

\section{INTRODUCTION}

Weighted Sobolev spaces are solution spaces of degenerate elliptic equations (see, for example, 1]). The type of a weight depends on the equation type. Similar to the classical theory of Sobolev spaces, embedding theorems of weighted Sobolev spaces are suitable for the corresponding elliptic boundary problems, especially for the existence and uniqueness of solutions. Embedding operators for weighted Sobolev spaces in smooth domains were studied by many authors (see, for example, [1]-[6]) with the help of the integral representations theory adopted to the weighted case. Weighted Sobolev spaces in nonsmooth domains were not studied before, except article [7, where some sufficient conditions for boundedness of the embedding operators were obtained. The main technical problem for the nonsmooth case is an adequate description of an interplay between weights and boundary types (singularities). The adequate choice allows us to obtain sharp Sobolev-type embeddings.

The relation between Jacobians of quasiconformal homeomorphisms and admissible weights for Sobolev and Poincaré inequalities was studied in [8]. In the present article we introduce a new approach based on the concept of 'generalized' quasiconformal homeomorphisms (or homeomorphisms with bounded mean distortion in another terminology) that induce bounded composition operators of weighted Sobolev spaces. These homeomorphisms transform the original embedding operators on nonsmooth domains to the embedding operators on smooth domains with a corresponding weight change. This approach was suggested in 9. for classical

Received by the editors April 23, 2007 and, in revised form, August 16, 2007.

2000 Mathematics Subject Classification. Primary 46E35, 30C65.

The second author was partially supported by the Israel Ministry of Immigrant Absorption.

(C)2009 American Mathematical Society

Reverts to public domain 28 years from publication 
Sobolev spaces on nonsmooth domains and can be briefly described with the help of the following diagram:

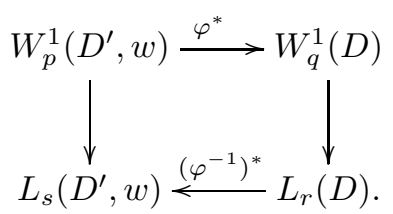

Here the operator $\varphi^{*} f=f \circ \varphi$ is a bounded composition operator of Sobolev spaces induced by a homeomorphism $\varphi$ that maps smooth domains $D \subset \mathbb{R}^{n}$ onto nonsmooth domains $D^{\prime} \subset \mathbb{R}^{n}$. Suppose that its inverse homeomorphism $\varphi^{-1}$ induces a bounded composition operator of corresponding Lebesgue spaces. If the Sobolev space $W_{q}^{1}(D)$ permits a bounded (compact) embedding operator into $L_{r}(D)$, then, using the corresponding compositions, we can construct the embedding operator of the weighted Sobolev space $W_{p}^{1}\left(D^{\prime}, w\right)$ into $L_{s}\left(D^{\prime}, w\right)$. The same scheme was used in the article 10] for the study of the embedding operators of $W_{2}^{1}$ into $L_{2}$ on nonsmooth bounded domains. In article [11 the same approach was applied to embedding problems for domains of Carnot groups.

Let us shortly describe the content of the paper. In section 1 we give necessary definitions and prove the density of smooth functions in weighted Sobolev spaces with weights satisfying the $A_{p}$-condition. Such weighted Sobolev spaces are Banach spaces. In section 2 we introduce classes of quasi-isometrical homeomorphisms and prove sufficient conditions for the compactness of the embedding operators for the weighted Sobolev spaces in domains quasi-isometrically equivalent to smooth ones. In section 3 we introduce classes of homeomorphisms with bounded mean distortion and study embedding operators for weighted Sobolev spaces defined on images of smooth domains. We apply these abstract results to domains with anisotropic Hölder-type singularities. The obtained estimates are sharper than the known result 7 .

In section 5 we apply embedding theorems for weighted Sobolev spaces to degenerate elliptic boundary problems.

\section{WEIGHTED SPACES}

In this paper we study weighted Lebesgue and Sobolev spaces defined in the domains of $n$-dimensional Euclidean space $\mathbb{R}^{n}, n \geq 2$.

Let $D$ be an open subset of $\mathbb{R}^{n}, n \geq 2$, and $w: \mathbb{R}^{n} \rightarrow[0, \infty)$ be a locally summable nonnegative function, i.e., a weight. Define a weighted Lebesgue space $L_{p}(D, w), 1 \leq p<\infty$, as a Banach space of locally summable functions $f: D \rightarrow \mathbb{R}$ equipped with the following norm:

$$
\left\|f \mid L_{p}(D, w)\right\|=\left(\int_{D}|f|^{p}(x) w(x) d x\right)^{1 / p}, 1 \leq p<\infty .
$$

Define a weighted Sobolev space $W_{p}^{m}(D, w), 1 \leq m<\infty, 1 \leq p<\infty$, as a normed space of locally summable, $m$ times weakly differentiable functions $f: D \rightarrow \mathbb{R}$ equipped with the following norm:

$$
\left\|f \mid W_{p}^{m}(D, w)\right\|=\left(\int_{D}|f|^{p}(x) w(x) d x\right)^{1 / p}+\sum_{|\alpha|=m}\left(\int_{D}\left|D^{\alpha} f\right|^{p}(x) w(x) d x\right)^{1 / p},
$$


where $\alpha:=\left(\alpha_{1}, \alpha_{2}, \ldots, \alpha_{n}\right)$ is a multi-index, $\alpha_{i}=0,1, \ldots,|\alpha|=\alpha_{1}+\alpha_{2}+\ldots+\alpha_{n}$ and $D^{\alpha} f$ is the weak derivative of order $\alpha$ of the function $f$ :

$$
\int_{D} f D^{\alpha} \eta d x=(-1)^{|\alpha|} \int_{D}\left(D^{\alpha} f\right) \eta d x, \quad \forall \eta \in C_{0}^{\infty}(D) .
$$

As usual $C_{0}^{\infty}(D)$ is the space of infinitely smooth functions with compact support.

By technical reasons we will need also a seminormed space $L_{p}^{m}(D)$ of locally summable, $m$ times weakly differentiable functions $f: D \rightarrow \mathbb{R}$ equipped with the following seminorm:

$$
\left\|f \mid L_{p}^{1}(D, w)\right\|=\sum_{|\alpha|=m}\left(\int_{D}\left|D^{\alpha} f\right|^{p}(x) w(x) d x\right)^{1 / p} .
$$

Without additional restrictions the space $W_{p}^{m}(D, w)$ is not necessarily a Banach space (see, for example, [1]).

Let us assume additionally that the weight $w: \mathbb{R}^{n} \rightarrow[0, \infty)$ satisfies the wellknown $A_{p}$-condition:

$$
\sup _{B \subset \mathbb{R}^{n}}\left(\frac{1}{|B|} \int_{B} w\right)\left(\frac{1}{|B|} \int_{B} w^{1 /(1-p)}\right)^{p-1}<+\infty,
$$

where $1<p<\infty$, and $|B|$ is the Lebesgue measure of the ball $B$.

Theorem 1. Let $D \subset \mathbb{R}^{n}$ be an open set and let a weight $w$ satisfy the $A_{p}$-condition. Then $W_{p}^{m}(D, w), 1 \leq m<\infty, 1<p<\infty$, is a Banach space. Smooth functions of the class $W_{p}^{m}(D, w)$ are dense in $W_{p}^{m}(D, w)$.

Let us give some remarks before the proof.

Suppose that the nonnegative function $\omega: \mathbb{R}^{n} \rightarrow[0, \infty)$ belongs to $C^{\infty}\left(\mathbb{R}^{n}\right)$, $\operatorname{supp} \omega \subset \overline{B(0,1)}$ and

$$
\int_{\mathbb{R}^{n}} \omega d x=1
$$

Denote by

$$
A_{r} f(x)=\frac{1}{r^{n}} \int_{\mathbb{R}^{n}} \omega\left(\frac{x-z}{r}\right) f(z) d z
$$

a mollifier function of $f$ with a mollification kernel $\omega$.

Let $D_{\delta}=\{x \in D: \operatorname{dist}(x, \partial D)>\delta\}$ for $\delta>0$. The proof of the theorem is based on the following lemma (see, for example, [12]):

Lemma 1. Let $D \subset \mathbb{R}^{n}$ be an open set and let a function $f \in L_{1, \mathrm{loc}}(D)$ have a weak derivative $D^{\alpha} f$ on $D$. Then for every $0<r<\delta$,

$$
D^{\alpha}\left(A_{r} f\right)=A_{r}\left(D^{\alpha} f\right) \text { on } D_{\delta} .
$$

Proof. For the reader's convenience we reproduce here a version of the proof. Note, that for every $x \in D_{\delta}$,

$$
\left(A_{r} f\right)(x)=\int_{B(0,1)} f(x-r z) \omega(z) d z, \quad 0<r<\delta .
$$

By definition of the weak derivative, $D_{x}^{\alpha}(f(x-r z))=\left(D_{x}^{\alpha} f\right)(x-r z)$ on $D_{\delta}$. 
Suppose $(x, z) \in D_{\delta} \times B(0,1)$. Define $F(x, z)=f(x-r z) \omega(z)$ and $G(x, z)=$ $\left(D_{x}^{\alpha} f\right)(x-r z) \omega(z)$. Then for each compact $K \subset D_{\delta}$ the functions $F, G$ belong to $L_{1}(K \times B(0,1))$. Moreover, by Fubini's theorem and the definition of the mollification kernel, we get

$$
\begin{gathered}
\int_{K}\left(\int_{B(0,1)}|f(x-r z) \omega(z)| d z\right) d x=\int_{K}\left(\frac{1}{r^{n}} \int_{B(x, r)}\left|f(y) \omega\left(\frac{x-y}{r}\right)\right| d y\right) d x \\
=\int_{K^{r}}\left(\frac{1}{r^{n}} \int_{K}\left|f(y) \omega\left(\frac{x-y}{r}\right)\right| d x\right) d y \leq \int_{K^{r}}|f(y)|\left(\frac{1}{r^{n}} \int_{K} \omega\left(\frac{x-y}{r}\right) d x\right) d y \\
\quad \leq \int_{K^{r}}|f(y)|\left(\frac{1}{r^{n}} \int_{\mathbb{R}^{n}} \omega\left(\frac{x-y}{r}\right) d x\right) d y=\int_{K^{r}}|f(y)| d y=\left\|f \mid L_{1}\left(K^{r}\right)\right\| .
\end{gathered}
$$

Here $K^{r}$ is the $r$-neighborhood of $K, \overline{K^{r}} \subset D$. Of course, the same estimate is correct for $G$.

Using the Fubini theorem we have

$$
\begin{array}{r}
\int_{D_{\delta}}\left(\int_{B(0,1)} D_{x}^{\alpha} f(x-r z) \omega(z) d z\right) \eta(x) d x=\int_{B(0,1)}\left(\int_{D_{\delta}}\left(D_{x}^{\alpha} f(x-r z) \omega(z)\right) \eta(x) d x\right) d z \\
=(-1)^{|\alpha|} \int_{B(0,1)}\left(\int_{D_{\delta}} f(x-r z) \omega(z)\left(D^{\alpha} \eta(x)\right) d x\right) d z \\
=(-1)^{|\alpha|} \int_{D_{\delta}}\left(\int_{B(0,1)} f(x-r z) \omega(z) d z\right) D^{\alpha} \eta(x) d x
\end{array}
$$

for every function $\eta \in C_{0}^{\infty}\left(D_{\delta}\right)$.

Hence for every $x \in D_{\delta}$,

$$
\begin{aligned}
D^{\alpha}\left(\left(A_{r} f\right)(x)\right)=D^{\alpha}\left(\int_{B(0,1)} f(x-r z) \omega(z) d z\right) \\
=\int_{B(0,1)} D_{x}^{\alpha} f(x-r z) \omega(z) d z=\left(A_{r}\left(D^{\alpha} f\right)\right)(x) .
\end{aligned}
$$

Proof of Theorem 1. Fix $\delta>0$. Since the weight $w$ satisfies the $A_{p}$-condition, the Hardy-Littlewood maximal operator

$$
M f(x)=\sup _{\delta>r>0} \frac{1}{r^{n}} \int_{B(x, r)} f(z) d z
$$


is bounded in $L_{p}\left(D_{\delta}, w\right)$ [13. Hence

$$
\begin{gathered}
\left\|A_{r} f-f \mid L_{p}\left(D_{\delta}, w\right)\right\|=\left(\int_{D_{\delta}}\left|\int_{B(0,1)} f(x-r z) \omega(z) d z-f(x)\right|^{p} w(x) d x\right)^{\frac{1}{p}} \\
\left.=\left.\left(\int_{D_{\delta}} \mid \int_{B(0,1)}(f(x-r z)-f(x)) \omega(z) d z\right)\right|^{p} w(x) d x\right)^{\frac{1}{p}} \\
\leq\|M\| \max _{x \in B(0,1)} \omega(x)\left(\int_{D_{\delta}}|f(x-r z)-f(x)|^{p} w(x) d x\right)^{\frac{1}{p}} .
\end{gathered}
$$

Here $\|M\|$ is the norm of the maximal operator in the space $L_{p}\left(D_{\delta}, w\right)$.

From the last inequality it follows, that for continuous functions $f$,

$$
A_{r} f \rightarrow f \quad \text { in } L_{p}\left(D_{\delta}, w\right) .
$$

Using an approximation of an arbitrary function $f \in L_{p}\left(D_{\delta}, w\right)$ by continuous functions (see, for example, [14]) the convergence can by obtained for $f \in L_{p}\left(D_{\delta}, w\right)$ also.

By Lemma 1,

$$
A_{r} f \rightarrow f \quad \text { in } \quad W_{p}^{m}\left(D_{\delta}, w\right)
$$

for an arbitrary function $f \in W_{p}^{m}(D, w)$. Therefore smooth functions are dense in $W_{p}^{m}\left(D_{\delta}, w\right)$.

The density of smooth functions of class $W_{p}^{m}(D, w)$ in $W_{p}^{m}(D, w)$ will be proved using the scheme proposed in 15 .

Choose a sequence of open sets $D_{j} \Subset D_{j+1} \Subset D, j \geq 1$, compactly embedded one into another, such that $\bigcup_{j} D_{j}=D$. Let $\Psi$ be a partition of unity on $D$, subordinate to the covering $D_{j+1} \backslash \bar{D}_{j-1}$. Let $\psi_{j}$ denote the (finite) sum of those $\psi \in \Psi$ for which supp $\psi \subset D_{j+1} \backslash \bar{D}_{j-1}$. Thus $\psi_{j} \in C_{0}^{\infty}\left(D_{j+1} \backslash \bar{D}_{j-1}\right)$ and $\sum_{j} \psi_{j} \equiv 1$ in $D$.

Fix $\varepsilon>0$ and for each $j=1,2, \ldots$ choose $\varphi_{j} \in C_{0}^{\infty}\left(D_{j+1} \backslash \bar{D}_{j-1}\right)$ such that

$$
\left\|\varphi_{j}-\psi_{j} f \mid W_{p}^{m}(D, w)\right\| \leq \varepsilon 2^{-j} .
$$

Then $\varphi=\sum_{j} \varphi_{j} \in C^{\infty}(D)$ and

$$
\begin{aligned}
\left\|\varphi-f \mid W_{p}^{m}(D, w)\right\|=\| \sum_{j} \varphi_{j}-\sum_{j} \psi_{j} f & \mid W_{p}^{m}(D, w) \| \\
& \leq \sum_{j}\left\|\varphi_{j}-\psi_{j} f \mid W_{p}^{m}(D, w)\right\|<\varepsilon .
\end{aligned}
$$

Therefore, the weighted space $W_{p}^{m}(D, w)$ is a Banach space and smooth functions of the class $W_{p}^{m}(D, w)$ are dense in this space.

Denote by $w(A)=\int_{A} w(x) d x$ the weighted measure (the measure associated with the weight $w$ ) of a measurable set $A \subset \mathbb{R}^{n}$. By 13 the Muckenhoupt $A_{p}$-condition leads to the doubling condition for the weighted measure; i.e., $\mu(B(x, 2 r)) \leq C \alpha \mu(B(x, r))$ for all $x \in \mathbb{R}^{n}$ and $r>0$. 
Definition 1. We call a bounded subdomain $D$ of Euclidean space $\mathbb{R}^{n}$ an embedding domain if for any function $f \in L_{q}^{1}(D), 1 \leq q<n$, the Sobolev-Poincaré-type inequality

$$
\inf _{c \in \mathbb{R}}\left\|f-c\left|L_{r}(D)\|\leq M\| f\right| L_{q}^{1}(D)\right\|
$$

holds for any $r \leq n q /(n-q)$. Here a constant $M$ depends on $q$ and $r$ only.

Recall that for any embedding domain and for any $r<n q /(n-q)$ the corresponding embedding operator $W_{q}^{1}(D) \hookrightarrow L_{r}(D)$ is compact.

Lipschitz bounded domains $D \subset \mathbb{R}^{n}$ represent examples of embedding domains. Let us recall also that Sobolev-type embeddings for smooth domains have been studied thoroughly and a discussion about the different aspects of the embedding problem can be found, for example, in [16].

\section{Quasi-ISOMetrical mappings And Sobolev Embeddings}

Let $D$ and $D^{\prime}$ be domains in Euclidean space $\mathbb{R}^{n}, n \geq 2$. A homeomorphism $\varphi: D \rightarrow D^{\prime}$ is called $Q$-quasi-isometrical if there exists a constant $0<Q<+\infty$, such that

for all points $x \in D$. Here

$$
\frac{1}{Q} \leq \underline{\varphi}^{\prime}(x) \leq \bar{\varphi}^{\prime}(x) \leq Q
$$

$$
\underline{\varphi}^{\prime}(x)=\liminf _{z \rightarrow x} \frac{|\varphi(x)-\varphi(z)|}{|x-z|} \text { and } \quad \bar{\varphi}^{\prime}(x)=\limsup _{z \rightarrow x} \frac{|\varphi(x)-\varphi(z)|}{|x-z|} .
$$

It is well known that any $Q$-quasi-isometrical homeomorphism is locally biLipschitz, weakly differentiable and differentiable almost everywhere in $D$. Hence its Jacobi matrix $D \varphi=\left(\frac{\partial \varphi_{i}}{\partial x_{j}}\right), i, j=1, \ldots, n$ and its Jacobian $J(x, \varphi)=\operatorname{det}\left(\frac{\partial \varphi_{i}}{\partial x_{j}}\right)$ are well defined almost everywhere in $D$. By definition of a $Q$-quasi-isometrical homeomorphism,

$$
Q^{-n} \leq|J(x, \varphi)| \leq Q^{n}
$$

almost everywhere.

Let us recall also that a quasi-isometrical homeomorphism has the Luzin $N$ property: the image of a set of measure zero is a set of measure zero.

Therefore, for any $Q$-quasi-isometrical homeomorphism $\varphi$, the change of variable formula in the Lebesgue integral

$$
\int_{\varphi(E)} f(y) d y=\int_{E} f \circ \varphi(x)|J(x, \varphi)| d x
$$

holds for any nonnegative measurable function $f$ and any measurable set $E \subset D$ [17.

Suppose weight $w$ satisfies the $A_{p}$-condition and that a homeomorphism $\varphi$ : $\mathbb{R}^{n} \rightarrow \mathbb{R}^{n}$ is $Q$-quasi-isometrical. Combining the change of variable formula and the two-side estimate for $|J(x, \varphi)|$ we can conclude that the weight $w \circ \varphi$ also satisfies the $A_{p}$-condition.

Theorem 2. Let $\varphi: D \rightarrow D^{\prime}$ be a Q-quasi-isometrical homeomorphism. Then a composition operator $\varphi^{*} f=f \circ \varphi$ is an isomorphism of the Sobolev spaces $W_{p}^{1}(D, w)$ and $W_{p}^{1}\left(D^{\prime}, w^{\prime}\right), 1<p<\infty$, where $w=w^{\prime} \circ \varphi$. 
Proof. Choose an arbitrary function $f \in W_{p}^{1}\left(D^{\prime}, w^{\prime}\right)$. By [14, $f$ belongs to the space $W_{1, \text { loc }}^{1}\left(D^{\prime}\right)$ and by [17] the composition $f \circ \varphi \in W_{1, \text { loc }}^{1}(D)$. Hence

$$
\begin{gathered}
\left\|\varphi^{*} f \mid W_{p}^{1}(D, w)\right\|=\left(\int_{D}|f \circ \varphi|^{p}\left(w^{\prime} \circ \varphi\right)(x) d x\right)^{\frac{1}{p}}+\left(\int_{D}|\nabla(f \circ \varphi)|^{p}\left(w^{\prime} \circ \varphi\right)(x) d x\right)^{\frac{1}{p}} \\
\leq\left(\int_{D}|f \circ \varphi|^{p}\left(w^{\prime} \circ \varphi\right)(x)|J(x, \varphi)| \frac{1}{|J(x, \varphi)|} d x\right)^{\frac{1}{p}} \\
+\left(\int_{D}|\nabla f|^{p}(\varphi(x))\left(w^{\prime} \circ \varphi\right)(x)|J(x, \varphi)| \frac{\left|\bar{\varphi}^{\prime}(x)\right|^{p}}{|J(x, \varphi)|} d x\right)^{\frac{1}{p}}
\end{gathered}
$$

Since $\varphi$ is a $Q$-quasi-isometrical homeomorphism the following estimates are correct:

$$
\frac{1}{|J(x, \varphi)|} \leq Q^{n} \quad \text { for almost all } x \in D
$$

and

$$
\left|\bar{\varphi}^{\prime}(x)\right| \leq Q \quad \text { for almost all } x \in D
$$

Hence

$$
\begin{aligned}
\left\|\varphi^{*} f \mid W_{p}^{1}(D, w)\right\| \leq Q^{\frac{n}{p}}\left(\int_{D}|f \circ \varphi|^{p}\left(w^{\prime} \circ \varphi\right)(x)|J(x, \varphi)| d x\right)^{\frac{1}{p}} & \\
& +Q^{\frac{n}{p}+1}\left(\int_{D}|\nabla f|^{p}(\varphi(x)) w^{\prime} \circ \varphi(x)|J(x, \varphi)| d x\right)^{\frac{1}{p}} .
\end{aligned}
$$

Using the change of variable formula we finally get the following inequality:

$$
\begin{aligned}
\left\|\varphi^{*} f \mid W_{p}^{1}(D, w)\right\| \leq Q^{\frac{n}{p}}\left(\int_{D^{\prime}}|f|^{p} w^{\prime}(y) \mid d y\right)^{\frac{1}{p}} & +Q^{\frac{n}{p}+1}\left(\int_{D^{\prime}}|\nabla f|^{p}(y) w^{\prime}(y) \mid d y\right)^{\frac{1}{p}} \\
& \leq Q^{\frac{n}{p}}(Q+1)\left\|f \mid W_{p}^{1}\left(D^{\prime}, w^{\prime}\right)\right\| .
\end{aligned}
$$

Since the inverse homeomorphism $\varphi^{-1}$ is also a $Q$-quasi-isometrical one, the inverse inequality

$$
\left\|\left(\varphi^{-1}\right)^{*} g\left|W_{p}^{1}\left(D^{\prime}, w^{\prime}\right)\left\|\leq Q^{\frac{n}{p}}(Q+1)\right\| g\right| W_{p}^{1}(D, w)\right\|, \quad g \in W_{p}^{1}(D, w),
$$

is also correct.

Corollary 1. Let $D$ and $D^{\prime}$ be domains in the Euclidean space $\mathbb{R}^{n}$. Suppose there exists a Q-quasi-isometrical homeomorphism $\varphi: D \rightarrow D^{\prime}$. Then the embedding operator

$$
i: W_{p}^{1}(D, w) \hookrightarrow L_{r}(D, w)
$$

is bounded (compact) if and only if the embedding operator

$$
i^{\prime}: W_{p}^{1}\left(D^{\prime}, w^{\prime}\right) \hookrightarrow L_{r}\left(D^{\prime}, w^{\prime}\right)
$$

is bounded (compact). 
Proof. Suppose the embedding operator

$$
i: W_{p}^{1}(D, w) \hookrightarrow L_{r}(D, w)
$$

is bounded (compact). Since $\varphi^{-1}$ is a $Q$-quasi-isometrical homeomorphism, then a composition operator $\left(\varphi^{-1}\right)^{*}=g \circ \varphi^{-1}: L_{r}(D, w) \hookrightarrow L_{r}\left(D^{\prime}, w^{\prime}\right)$ is bounded, as one can see by a simple calculation:

$$
\begin{aligned}
& \left\|\left(\varphi^{-1}\right)^{*} g \mid L_{r}\left(D^{\prime}, w^{\prime}\right)\right\| \\
& =\left(\int_{D^{\prime}}\left|g \circ \varphi^{-1}\right|^{r}(y) w^{\prime}(y) d y\right)^{\frac{1}{r}}=\left(\int_{D}|g|^{r}(x) w^{\prime} \circ \varphi(x)|J(x, \varphi)| d x\right)^{\frac{1}{r}} \\
& \leq Q^{\frac{n}{r}}\left(\int_{D}|g|^{r}(x) w(x) d x\right)^{\frac{1}{r}}=Q^{\frac{n}{r}}\left\|g \mid L_{r}(D, w)\right\| .
\end{aligned}
$$

Therefore, the embedding operator $i^{\prime}: W_{p}^{1}\left(D^{\prime}, w^{\prime}\right) \hookrightarrow L_{r}\left(D^{\prime}, w^{\prime}\right)$ is bounded (compact) as a composition of bounded operators $\varphi^{*},\left(\varphi^{-1}\right)^{*}$ and a bounded (compact) embedding operator $W_{p}^{1}(D, w) \hookrightarrow L_{r}(D, w)$.

The proof in the inverse direction is the same.

By Corollary 1 an image $D^{\prime}=\varphi(D)$ of an embedding domain $D$ under a quasiisometrical homeomorphism $\varphi$ is an embedding domain also. In the paper [10] the various examples of embedding domains of such a type were discussed.

The next theorem demonstrates simple conditions for compactness of the embedding operators of weighted Sobolev spaces.

Theorem 3. Let $D^{\prime} \subset \mathbb{R}^{n}$ be a quasi-isometrical image of an embedding domain $D, 1 \leq s \leq r<n q /(n-q), q \leq p, 1<p<\infty$, and

$$
K(w)=\max \left\{\left\|w^{-\frac{1}{p}}\left|L_{\frac{p q}{p-q}}\left(D^{\prime}\right)\|,\| w^{\frac{1}{s}}\right| L_{\frac{r s}{r-s}}\left(D^{\prime}\right)\right\|\right\}<+\infty .
$$

Then the embedding operator

$$
i: W_{p}^{1}\left(D^{\prime}, w\right) \hookrightarrow L_{s}\left(D^{\prime}, w\right)
$$

is a compact operator.

For $r=n q /(n-q)$ the embedding operator $i$ is bounded only.

Proof. By the conditions of the theorem, there exists a $Q$-quasi-isometrical homeomorphism $\varphi: D \rightarrow D^{\prime}$ of the embedding domain $D$ onto the domain $D^{\prime}$. For any function $u \in W_{p}^{1}\left(D^{\prime}, w\right)$ the composition $u \circ \varphi$ is weakly differentiable in the domain $D$, and the following estimate is correct:

$$
\begin{aligned}
\left\|u \circ \varphi \mid W_{q}^{1}(D)\right\| & =\left(\int_{D}|u \circ \varphi|^{q} d x\right)^{\frac{1}{q}}+\left(\int_{D}|\nabla(u \circ \varphi)|^{q} d x\right)^{\frac{1}{q}} \\
\leq & \left(\int_{D}|u \circ \varphi|^{q}\left(|J(x, \varphi)| w(\varphi(x))^{\frac{q}{p}} \frac{1}{\left(|J(x, \varphi)| w(\varphi(x))^{\frac{q}{p}}\right.} d x\right)^{\frac{1}{q}}\right. \\
& +\left(\int_{D}|\nabla u|^{q}\left|\bar{\varphi}^{\prime}(x)\right|^{q}\left(|J(x, \varphi)| w(\varphi(x))^{\frac{q}{p}} \frac{1}{\left(|J(x, \varphi)| w(\varphi(x))^{\frac{q}{p}}\right.} d x\right)^{\frac{1}{q}} .\right.
\end{aligned}
$$


By the Hölder inequality,

$$
\begin{aligned}
& \left\|u \circ \varphi \mid W_{q}^{1}(D)\right\| \\
& \leq\left(\int_{D}\left(\frac{1}{|J(x, \varphi)| w(\varphi(x))}\right)^{\frac{q}{p-q}} d x\right)^{\frac{p-q}{p q}}\left(\int_{D}|u|^{p}(\varphi(x)) w(\varphi(x))|J(x, \varphi)| d x\right)^{\frac{1}{p}} \\
& +\left(\int_{D}\left(\frac{\left|\bar{\varphi}^{\prime}(x)\right|^{p}}{|J(x, \varphi)| w(\varphi(x))}\right)^{\frac{q}{p-q}} d x\right)^{\frac{p-q}{p q}}\left(\int_{D}|\nabla u|^{p}(\varphi(x)) w(\varphi(x))|J(x, \varphi)| d x\right)^{\frac{1}{p}} .
\end{aligned}
$$

Since $\varphi$ is a $Q$-quasi-isometrical homeomorphism, then by the change of variable formula for the Lebesgue integral we obtain

$$
\begin{aligned}
&\left\|u \circ \varphi \mid W_{q}^{1}(D)\right\| \leq Q^{\frac{n}{q}}\left(\int_{D^{\prime}} w(y)^{\frac{q}{q-p}} d y\right)^{\frac{p-q}{p q}}\left(\int_{D^{\prime}}|u|^{p} w(y) d y\right)^{\frac{1}{p}} \\
&+Q^{\frac{p-q+n}{q}}\left(\int_{D^{\prime}} w(y)^{\frac{q}{q-p}} d y\right)^{\frac{p-q}{p q}}\left(\int_{D^{\prime}}|\nabla u|^{p} w(y) d y\right)^{\frac{1}{p}} \\
& \leq Q^{\frac{n}{q}} K(w)\left\|u\left|L_{p}\left(D^{\prime}, w\right)\left\|+Q^{\frac{p-q+n}{q}} K(w)\right\| \nabla u\right| L_{p}\left(D^{\prime} w\right)\right\| .
\end{aligned}
$$

By the previous inequality the composition operator

$$
\varphi^{*}: W_{p}^{1}\left(D^{\prime}, w\right) \rightarrow W_{q}^{1}(D), 1 \leq q \leq p<+\infty,
$$

is bounded.

Let us prove boundedness of the composition operator $\left(\varphi^{-1}\right)^{*}: L_{r}(D) \rightarrow$ $L_{s}\left(D^{\prime}, w\right)$. By the theorem's conditions the quantity $\left\|w^{\frac{1}{s}} \mid L_{\frac{r s}{r-s}}\left(D^{\prime}\right)\right\|$ is finite. Hence by [18] the composition operator

$$
\left(\varphi^{-1}\right)^{*}: L_{r}(D) \rightarrow L_{s}\left(D^{\prime}, w\right), 1 \leq s \leq r<+\infty,
$$

is bounded.

Because $D$ is an embedding domain the embedding operator $i: W_{q}^{1}(D) \hookrightarrow L_{r}(D)$ is compact for any $r<n q /(n-q)$ and bounded for $r=n q /(n-q)$. Therefore the embedding operator $i^{\prime}: W_{p}^{1}\left(D^{\prime}, w\right) \hookrightarrow L_{s}\left(D^{\prime}, w\right)$ is compact (bounded) as a composition of bounded operators $\varphi^{*},\left(\varphi^{-1}\right)^{*}$ and the compact (bounded) embedding operator $i$ for any $r<n q /(n-q)(r=n q /(n-q))$.

In a similar way we can prove

Theorem 4. Let $D^{\prime} \subset \mathbb{R}^{n}$ be a quasi-isometrical image of an embedding domain $D, 1 \leq s \leq r<n q /(n-q), q \leq p, 1<p<\infty$, and

$$
K(w)=\left\|w^{\frac{1}{s}} \mid L_{\frac{r s}{r-s}}\left(D^{\prime}\right)\right\|<+\infty .
$$

Then the embedding operator

$$
i: W_{p}^{1}\left(D^{\prime}\right) \hookrightarrow L_{s}\left(D^{\prime}, w\right)
$$

is compact.

For $r=n q /(n-q)$ the embedding operator $i$ is bounded only.

The next lemma allows us to construct various examples of the embedding domains. 
Lemma 2. Let $D_{1}$ and $D_{2}$ be domains such that the embedding operators

$$
\begin{aligned}
& i: W_{p}^{1}\left(D_{1}, w\right) \hookrightarrow L_{s}\left(D_{1}, v\right), \\
& i: W_{p}^{1}\left(D_{2}, w\right) \hookrightarrow L_{s}\left(D_{2}, v\right)
\end{aligned}
$$

are compact. Then the embedding operator

$$
i: W_{p}^{1}\left(D_{1} \cup D_{2}, w\right) \hookrightarrow L_{s}\left(D_{1} \cup D_{2}, v\right)
$$

is also compact.

Proof. We prove this lemma by the scheme suggested in 10 . Choose a sequence of functions $\left\{f_{n}\right\} \subset W_{p}^{1}\left(D_{1} \cup D_{2}, w\right)$ such that $\left\|f_{n} \mid W_{p}^{1}\left(D_{1} \cup D_{2}, w\right)\right\| \leq 1$ for all $n$. Let $g_{n}=\left.f_{n}\right|_{D_{1}}$ and $h_{n}=\left.f_{n}\right|_{D_{2}}$. Then $g_{n} \in W_{p}^{1}\left(D_{1}\right), h_{n} \in W_{p}^{1}\left(D_{2}\right)$, $\left\|f_{n}\left|W_{p}^{1}\left(D_{1}\right)\|\leq 1,\| h_{n}\right| W_{p}^{1}\left(D_{2}\right)\right\| \leq 1$.

Because the embedding operator $i: W_{p}^{1}\left(D_{1}, w\right) \hookrightarrow L_{s}\left(D_{1}, v\right)$ is compact, we can choose a subsequence $\left\{g_{n_{k}}\right\}$ of the sequence $\left\{g_{n}\right\}$ which converges in $L_{s}\left(D_{1}, v\right)$ to a function $g_{0} \in L_{s}\left(D_{1}, v\right)$. Because the second embedding operator $i: W_{p}^{1}\left(D_{2}, w\right) \hookrightarrow$ $L_{s}\left(D_{2}, v\right)$ is also compact we can choose a subsequence $\left\{h_{n_{k_{m}}}\right\}$ of the sequence $\left\{h_{n_{k}}\right\}$ which converges in $L_{s}\left(D_{2}, v\right)$ to a function $h_{0} \in L_{s}\left(D_{2}, v\right)$. It is evident that $g_{0}=h_{0} v$-almost everywhere in $D_{1} \cap D_{2}$ and the function $f_{0}=g_{0}$ on $D_{1}$ and $f_{0}=h_{0}$ on $D_{2}$ belongs to $L_{s}\left(D_{1} \cup D_{2}, v\right)$.

Hence

$\left\|f_{n_{k_{m}}}-f_{0}\left|L_{s}\left(D_{1} \cup D_{2}, v\right)\|\leq\| g_{n_{k_{m}}}-g_{0}\right| L_{s}\left(D_{1}, v\right)\right\|+\left\|h_{n_{k_{m}}}-h_{0} \mid L_{s}\left(D_{2}, v\right)\right\|$.

Therefore $\left\|f_{n_{k_{m}}}-f_{0} \mid L_{s}\left(D_{1} \cup D_{2}, v\right)\right\| \longrightarrow 0$ for $m \longrightarrow \infty$.

\section{EMBEDDING OPERATORS FOR GENERAL DOMAINS}

Let $D$ and $D^{\prime}$ be domains in Euclidean space $\mathbb{R}^{n}, n \geq 2$. Remember that a homeomorphism $\varphi: D \rightarrow D^{\prime}$ belongs to the Sobolev class $W_{1, \text { loc }}^{1}(D)$ if its coordinate functions belong to $W_{1, \text { loc }}^{1}(D)$. Denote by $D \varphi$ the weak differential of $\varphi$. The norm $|D \varphi(x)|$ is the standard norm of the linear operator defined by $D \varphi(x)$.

Call a homeomorphism $\varphi: D \rightarrow D^{\prime} w$-weighted $(p, q)$-quasiconformal if $\varphi$ belongs to the Sobolev space $W_{1, \text { loc }}^{1}(D),|D \varphi|=0$ almost everywhere on the set $Z=\{x$ : $|J(x, \varphi)| w(\varphi(x))=0\}$ and the following inequality

$$
K_{p, q}(D, w)=\left[\int_{D}\left(\frac{|D \varphi(x)|^{p}}{|J(x, \varphi)| w(\varphi(x))}\right)^{\frac{q}{p-q}} d x\right]^{\frac{p-q}{p q}}<\infty
$$

is correct.

For $w \equiv 1$ we call a 1-weighted $(p, q)$-quasiconformal homeomorphism a $(p, q)$ quasiconformal one.

The following result was proved in [19] for a more general class of mappings. For the reader's convenience we reproduce here a simple version of the proof adopted to homeomorphisms.

Proposition 1. Let $D$ and $D^{\prime}$ be domains in Euclidean space $\mathbb{R}^{n}, n \geq 2$ and let $\varphi: D \rightarrow D^{\prime}$ be a w-weighted $(p, q)$-quasiconformal homeomorphism. Then a composition operator

$$
\varphi^{*}: L_{p}^{1}\left(D^{\prime}, w\right) \rightarrow L_{q}^{1}(D), \quad 1 \leq q \leq p<+\infty,
$$

is bounded. 
Proof. Let $f \in L_{p}^{1}\left(D^{\prime}, w\right)$ be a smooth function. Then $f \circ \varphi \in L_{1, \text { loc }}^{1}(D)$ and the following inequalities

$$
\begin{aligned}
\left\|\varphi^{*} f \mid L_{q}^{1}(D)\right\| & =\left(\int_{D}|\nabla(f \circ \varphi)|^{q} d x\right)^{\frac{1}{q}} \leq\left(\int_{D}|D \varphi|^{q}|\nabla f|^{q} d x\right)^{\frac{1}{q}} \\
& \leq\left(\int_{D}|D \varphi|^{q} \frac{1}{|J(x, \varphi)|^{\frac{q}{p}} w(\varphi(x))^{\frac{q}{p}}}|\nabla f|^{q}|J(x, \varphi)|^{\frac{q}{p}} w(\varphi(x))^{\frac{q}{p}} d x\right)^{\frac{1}{q}}
\end{aligned}
$$

are correct.

Using the Hölder inequality and the change of variable formula for the Lebesgue integral, we obtain

$$
\begin{aligned}
\| \varphi^{*} f & \mid L_{q}^{1}(D) \| \\
& \leq\left[\int_{D}\left(\frac{|D \varphi(x)|^{p}}{|J(x, \varphi)| w(\varphi(x))}\right)^{\frac{q}{p-q}} d x\right]^{\frac{p-q}{p q}}\left(\int_{D}|\nabla f|^{p}|J(x, \varphi)| w(\varphi(x)) d x\right)^{\frac{1}{p}} \\
& =K_{p, q}(D, w)\left\|f \mid L_{p}^{1}\left(D^{\prime}, w\right)\right\| .
\end{aligned}
$$

The fulfillment of the last inequality for an arbitrary function $f \in L_{p}^{1}\left(D^{\prime}, w\right)$ can be proved by an approximation of $f$ by smooth functions [19].

The next theorem gives a sufficient condition for the boundedness (compactness) of embedding operators in nonsmooth domains.

Theorem 5. Let a domain $D \subset \mathbb{R}^{n}$ be an embedding domain and let there exist a w-weighted $(p, q)$-quasiconformal homeomorphism $\varphi: D \rightarrow D^{\prime}$ of the domain $D$ onto the bounded domain $D^{\prime}$.

If for some $p \leq s \leq r<\infty$ the following inequality is correct,

$$
\int_{D}(|J(x, \varphi)| w(\varphi(x)))^{\frac{r}{r-s}} d x<+\infty,
$$

then an embedding operator

$$
i: W_{p}^{1}\left(D^{\prime}, w\right) \hookrightarrow L_{s}\left(D^{\prime}, w\right)
$$

is bounded, if $s \leq r \leq n q /(n-q)$, and is compact if $s \leq r<n q /(n-q)$.

Proof. Because

$$
\int_{D}(|J(x, \varphi)| w(\varphi(x)))^{\frac{r}{r-s}}<+\infty,
$$

the composition operator $\left(\varphi^{-1}\right)^{*}: L_{r}(D) \rightarrow L_{s}\left(D^{\prime}, w\right)$ is bounded; i.e., the following inequality

$$
\left\|\left(\varphi^{-1}\right)^{*} v\left|L_{s}\left(D^{\prime}, w\right)\left\|\leq A_{r, s}(D, w)\right\| v\right| L_{r}(D)\right\|
$$

is correct. Here $A_{r, s}(D, w)$ is a positive constant.

Because the domain $D$ is an embedding domain and the composition operators

$$
\left(\varphi^{-1}\right)^{*}: L_{r}(D) \rightarrow L_{s}\left(D^{\prime}, w\right), \varphi^{*}: L_{p}^{1}\left(D^{\prime}, w\right) \rightarrow L_{q}^{1}(D)
$$


are bounded, the following inequalities

$$
\begin{aligned}
\inf _{c \in \mathbb{R}} \| u- & c\left|L_{s}\left(D^{\prime}, w\right)\left\|\leq A_{r, s}(D, w) \inf _{c \in \mathbb{R}}\right\| v-c\right| L_{r}(D) \| \\
& \leq A_{r, s}(D, w) M\left\|v\left|L_{q}^{1}(D)\left\|\leq A_{r, s}(D, w) K_{p, q}(D, w) M\right\| u\right| L_{p}^{1}\left(D^{\prime}, w\right)\right\|
\end{aligned}
$$

hold. Here $M$ and $K_{p, q}(D, w)$ are positive constants.

The Hölder inequality implies the following estimate:

$$
\begin{aligned}
|c|=w\left(D^{\prime}\right)^{-\frac{1}{p}} \| c \mid & L_{p}\left(D^{\prime}, w\right) \| \leq w\left(D^{\prime}\right)^{-\frac{1}{p}}\left(\left\|u\left|L_{p}\left(D^{\prime}, w\right)\|+\| u-c\right| L_{p}\left(D^{\prime}, w\right)\right\|\right) \\
& \leq w\left(D^{\prime}\right)^{-\frac{1}{p}}\left\|u\left|L_{p}\left(D^{\prime}, w\right)\left\|+w\left(D^{\prime}\right)^{-\frac{1}{s}}\right\| u-c\right| L_{s}\left(D^{\prime}, w\right)\right\| .
\end{aligned}
$$

Because $q \leq r$ we have

$$
\begin{gathered}
\left\|v\left|L_{q}(D)\|\leq\| c\right| L_{q}(D)\right\|+\left\|v-c\left|L_{q}(D)\left\|\leq|c||D|^{\frac{1}{q}}+|D|^{\frac{r-q}{r}}\right\| v-c\right| L_{r}(D)\right\| \\
\leq\left(w\left(D^{\prime}\right)^{-\frac{1}{p}}\left\|u\left|L_{p}\left(D^{\prime}, w\right)\left\|+w\left(D^{\prime}\right)^{-\frac{1}{s}}\right\| u-c\right| L_{s}\left(D^{\prime}, w\right)\right\|\right)|D|^{\frac{1}{q}} \\
+|D|^{\frac{r-q}{r}}\left\|v-c \mid L_{r}(D)\right\| .
\end{gathered}
$$

From previous inequalities we obtain finally

$$
\begin{aligned}
\left\|v \mid L_{q}(D)\right\| & \leq|D|^{\frac{1}{q}} w\left(D^{\prime}\right)^{-\frac{1}{p}}\left\|u \mid L_{p}\left(D^{\prime}\right)\right\| \\
& +A_{r, s}(D, w) K_{p, q}(D, w) M|D|^{\frac{1}{q}} w\left(D^{\prime}\right)^{-\frac{1}{p}}\left\|u \mid L_{p}^{1}\left(D^{\prime}, w\right)\right\| \\
& +K_{p, q}(D, w) M|D|^{\frac{r-q}{r}}\left\|u \mid L_{p}^{1}\left(D^{\prime}, w\right)\right\| .
\end{aligned}
$$

Therefore the composition operator

$$
\varphi^{*}: W_{p}^{1}\left(D^{\prime}, w\right) \rightarrow W_{q}^{1}(D)
$$

is also bounded.

Finally we can conclude that the embedding operator $i: W_{p}^{1}\left(D^{\prime}, w\right) \hookrightarrow L_{s}\left(D^{\prime}, w\right)$ is bounded as the composition of bounded operators $\varphi^{*},\left(\varphi^{-1}\right)^{*}$ and the embedding operator $W_{q}^{1}(D) \hookrightarrow L_{r}(D)$ in the case $r \leq n q /(n-q)$. The embedding operator $i: W_{p}^{1}\left(D^{\prime}, w\right) \hookrightarrow L_{s}\left(D^{\prime}, w\right)$ is compact as the composition of bounded operators $\varphi^{*},\left(\varphi^{-1}\right)^{*}$ and the embedding operator $W_{q}^{1}(D) \hookrightarrow L_{r}(D)$ is compact in the case $r<n q /(n-q)$.

Let us apply these general results to domains with anisotropic Hölder singularities described in [9].

Let $g_{i}(\tau)=\tau^{\gamma_{i}}, \gamma_{i} \geq 1,0 \leq \tau \leq 1$. For the function $G=\prod_{i=1}^{n-1} g_{i}$ denote by

$$
\gamma=\frac{\log G(\tau)}{\log \tau}+1
$$

It is evident that $\gamma \geq n$. Let us consider the domain

$$
H_{g}=\left\{x \in \mathbb{R}^{n}: 0<x_{n}<1,0<x_{i}<g_{i}\left(x_{n}\right), i=1,2, \ldots, n-1\right\} .
$$

In the case $g_{1}=g_{2}=\cdots=g_{n-1}$ we will say that the domain $H_{g}$ is a domain with $\sigma$-Hölder singularity, $\sigma=(\gamma-1) /(n-1)$. For $g_{1}(\tau)=g_{2}(\tau)=\cdots=g_{n-1}(\tau)=\tau$ we will use the notation $H_{1}$ instead of $H_{g}$. The domain $H_{1}$ is quasi-isometrically homeomorphic to the standard unit ball. Hence by Theorem 3, domain $H_{1}$ is an embedding domain. 
If the weight is polynomial, i.e. $w(x):=|x|^{\alpha}$, then the $A_{p}$-condition is correct only if $-n<\alpha<n(p-1)$.

Theorem 6. Let $-n<\alpha<n(p-1)$ and $1<p<\alpha+\gamma$. Then the embedding operator

$$
W_{p}^{1}\left(H_{g},|x|^{\alpha}\right) \hookrightarrow L_{s}\left(H_{g},|x|^{\alpha}\right)
$$

is compact for any $1 \leq s<\frac{(\alpha+\gamma) p}{\alpha+\gamma-p}$.

Proof. For any $0<a<1$ we define a homeomorphism $\varphi_{a}: H_{1} \rightarrow H_{g}, a>0$, by the expression

$$
\varphi_{a}(x)=\left(\frac{x_{1}}{x_{n}} g_{1}^{a}\left(x_{n}\right), \ldots, \frac{x_{n-1}}{x_{n}} g_{n-1}^{a}\left(x_{n}\right), x_{n}^{a}\right)
$$

During the proof we will choose a number $a$ and a corresponding homeomorphism in such a way that the conditions of Theorem 5 will be fulfilled. By a simple calculation,

$$
\begin{aligned}
\frac{\partial\left(\varphi_{a}\right)_{i}}{\partial x_{i}}=\frac{g_{i}^{a}\left(x_{n}\right)}{x_{n}}, & \frac{\partial\left(\varphi_{a}\right)_{i}}{\partial x_{n}}=\frac{-x_{i} g_{i}^{a}\left(x_{n}\right)}{x_{n}^{2}}+\frac{a x_{i} g_{i}^{a-1}\left(x_{n}\right)}{x_{n}} g_{i}^{\prime}\left(x_{n}\right) \\
& \text { and } \frac{\partial\left(\varphi_{a}\right)_{n}}{\partial x_{n}}=a x_{n}^{a-1}
\end{aligned}
$$

for any $i=1, \ldots, n-1$.

Hence $J\left(x, \varphi_{a}(x)\right)=a x_{n}^{a-n} G^{a}\left(x_{n}\right)$. By definition, the functions $g_{i}, i=1,2, \ldots$, $n-1$ are Lipschitz functions. Therefore there exists a constant $M<+\infty$ such that

$$
g_{i}\left(x_{n}\right) \leq M x_{n} \quad \text { and } \quad g_{i}^{\prime}\left(x_{n}\right) \leq M
$$

for any $x_{n} \in[0,1]$ and $i=1,2, \ldots, n-1$. Using estimates for derivatives and the inequalities $x_{i} \leq x_{n}$ that are correct for all $x \in H_{1}$ we obtain the following estimate: $\left|D \varphi_{a}(x)\right| \leq c_{1} x_{n}^{a-1}$. By the same way we obtain also the two-sided estimate:

$$
c_{2} x_{n}^{\alpha a} \leq\left|\varphi_{a}(x)\right|^{\alpha} \leq c_{3} x_{n}^{\alpha a}
$$

Now we can check for which $q$ the homeomorphism $\varphi_{a}: H_{1} \rightarrow H_{g}$ is a $w$-weighted $(p, q)$-quasiconformal homeomorphism:

$$
\begin{gathered}
I_{a}=K_{p, q}\left(H_{1}, w\right)^{\frac{p q}{p-q}}=\int_{H_{1}}\left(\frac{\left|D \varphi_{a}(x)\right|^{p}}{\left|J\left(x, \varphi_{a}\right)\right| w\left(\varphi_{a}(x)\right)}\right)^{\frac{q}{p-q}} d x \\
\leq C \int_{0}^{1} \int_{0}^{x_{n}} \ldots \int_{0}^{x_{n}}\left(\frac{x_{n}^{p(a-1)}}{x_{n}^{a-n} G^{a}\left(x_{n}\right) x_{n}^{\alpha a}}\right)^{\frac{q}{p-q}} d x_{1} \ldots d x_{n-1} d x_{n} \\
\quad=C \int_{0}^{1} x_{n}^{(p(a-1)-a(\alpha+1)+n) \frac{q}{p-q}+n-1} G^{-a \frac{q}{p-q}}\left(x_{n}\right) d x_{n} .
\end{gathered}
$$

Hence the quantity $I_{a}$ is finite if

or

$$
(p(a-1)-a(\alpha+1)+n) \frac{q}{p-q}+n-a(\gamma-1) \frac{q}{p-q}>0
$$

$$
q<n p /(a(\alpha+\gamma)+p-a p)
$$

Hence, the homeomorphism $\varphi_{a}$ is a $w$-weighted $(p, q)$-quasiconformal homeomorphism. 
Let us check the conditions of Theorem 5. First we have to estimate the degree of integrability for the Jacobian $J_{a}$ of the homeomorphism $\varphi_{a}$ :

$$
\begin{aligned}
& J_{a}=\int_{H_{1}}(|J(x, \varphi)| w(\varphi(x)))^{\frac{r}{r-s}} d x \\
& \leq C \int_{0}^{1} \int_{0}^{x_{n}} \ldots \int_{0}^{x_{n}} x_{n}^{(a(\alpha+1)-n) \frac{r}{r-s}} G^{a \frac{r}{r-s}}\left(x_{n}\right) d x_{1} \ldots d x_{n-1} d x_{n} \\
& \leq C \int_{0}^{1} x_{n}^{(a(\alpha+1)-n) \frac{r}{r-s}+n-1+a \frac{r}{r-s}(\gamma-1)} d x_{n} .
\end{aligned}
$$

The integral $J_{a}$ converges if

$$
(a(\alpha+1)-n) \frac{r}{r-s}+n+a \frac{r}{r-s}(\gamma-1)>0,
$$

or

$$
s<\frac{a(\alpha+\gamma)}{n} r .
$$

Hence, the conditions of Theorem 5 are fulfilled if

$$
s<\frac{a(\alpha+\gamma)}{n} r, \quad r<\frac{n q}{n-q} \text { and } q<\frac{n p}{a(\alpha+\gamma)+p-a p} .
$$

Therefore

$$
s<\frac{a(\alpha+\gamma)}{n} \frac{n q}{n-q}<\frac{n p}{a(\alpha+\gamma-p)} \frac{a(\alpha+\gamma)}{n}=\frac{p(\alpha+\gamma)}{\alpha+\gamma-p} .
$$

Theorem 6 is proved.

Remark 1 . The conclusion of Theorem 6 is fulfilled for functions $g_{i}:[0,1] \rightarrow \mathbb{R}$, $i=1,2, \ldots, n-1$ such that

$$
C_{1} \tau^{\gamma_{i}} \leq g_{i}(\tau) \leq C_{2} \tau^{\gamma_{i}}
$$

for some constants $C_{1}$ and $C_{2}$.

From Theorem 6, Corollary 2 immediately follows.

Corollary 2. Let $D \subset \mathbb{R}^{n}$ be a domain with $\sigma$-Hölder singularity. Then the embedding operator

$$
W_{p}^{1}\left(D,|x|^{\alpha}\right) \hookrightarrow L_{s}\left(D,|x|^{\alpha}\right)
$$

is compact for

$$
s \leq \frac{(\sigma(n-1)+1+\alpha) p}{\sigma(n-1)+\alpha-(p-1)} .
$$

(Here $s \geq 0$, since $p<\alpha+\gamma, \sigma=(\gamma-1) /(n-1)$.)

Let us compare this result with the known ones. From the main result of [7] it follows that for an arbitrary domain $D$ with $\sigma$-Hölder singularity the embedding operator

$$
W_{p}^{1}\left(D,|x|^{\alpha}\right) \hookrightarrow L_{\tilde{s}}\left(D,|x|^{\alpha}\right)
$$


is bounded, while

$$
\tilde{s} \leq \frac{(n+\alpha) p}{\sigma(\alpha+n-1)-(p-1)} .
$$

Then $s>\tilde{s}$ while $\sigma>1$, and $s=\tilde{s}$ while $\sigma=1$. Hence our estimate is sharper.

The next results deal with embeddings of classical Sobolev spaces into weighted Lebesgue spaces.

Theorem 7. Let $D \subset \mathbb{R}^{n}$ be an embedding domain and let there exist a $(p, q)$ quasiconformal homeomorphism $\varphi$ of $D$ onto the bounded domain $D^{\prime}$.

If

$$
\int_{D}(|J(x, \varphi)| w(\varphi(x)))^{\frac{r}{r-s}}<+\infty,
$$

for a pair of numbers $p \leq s<r<\infty$, then an embedding operator

$$
i: W_{p}^{1}\left(D^{\prime}\right) \hookrightarrow L_{s}\left(D^{\prime}, w\right)
$$

is compact for $r<n q /(n-q)$ and is bounded for $r=n q /(n-q)$.

Proof. Because $D$ is an embedding domain, the $(p, q)$-quasiconformal homeomorphism $\varphi$ induces the bounded composition operator

$$
\varphi^{*}: W_{p}^{1}\left(D^{\prime}\right) \rightarrow W_{q}^{1}(D)
$$

(see [18]).

Because $\int_{D}(|J(x, \varphi)| w(\varphi(x)))^{\frac{r}{r-s}}<+\infty$ the composition operator for Lebesgue spaces

$$
\left\|\left(\varphi^{-1}\right)^{*} v\left|L_{s}\left(D^{\prime}, w\right)\left\|\leq A_{r, s}(D, w)\right\| v\right| L_{r}(D)\right\|
$$

is bounded also.

Finally we can conclude that

1) If $r \leq n q /(n-q)$, then the embedding operator $i: W_{p}^{1}\left(D^{\prime}\right) \hookrightarrow L_{s}\left(D^{\prime}, w\right)$ is bounded as a composition of bounded operators $\varphi^{*},\left(\varphi^{-1}\right)^{*}$ and the bounded embedding operator $W_{q}^{1}(D) \hookrightarrow L_{r}(D)$.

2) If $r<n q /(n-q)$, then the embedding operator $i: W_{p}^{1}\left(D^{\prime}\right) \hookrightarrow L_{s}\left(D^{\prime}, w\right)$ is compact as a composition of bounded operators $\varphi^{*},\left(\varphi^{-1}\right)^{*}$ and the compact embedding operator $W_{q}^{1}(D) \hookrightarrow L_{r}(D)$.

Apply the previous result to anisotropic Hölder domains.

Theorem 8. Let $1<p<\gamma$ and $1 \leq s<\frac{(\alpha+\gamma) p}{\gamma-p}$. Then the embedding operator

$$
W_{p}^{1}\left(H_{g}\right) \hookrightarrow L_{s}\left(H_{g},|x|^{\alpha}\right)
$$

is compact.

Proof. Similar to the proof of Theorem 6 , for any $0<a<1$, we define a homeomorphism $\varphi_{a}: H_{1} \rightarrow H_{g}, a>0$ by the expression

$$
\varphi_{a}(x)=\left(\frac{x_{1}}{x_{n}} g_{1}^{a}\left(x_{n}\right), \ldots, \frac{x_{n-1}}{x_{n}} g_{n-1}^{a}\left(x_{n}\right), x_{n}^{a}\right) .
$$

During the proof we will choose a number $a$ and the corresponding homeomorphism in such a way that the conditions of Theorem 7 will be fulfilled. 
By a simple calculation we have

$$
\begin{aligned}
& \frac{\partial\left(\varphi_{a}\right)_{i}}{\partial x_{i}}=\frac{g_{i}^{a}\left(x_{n}\right)}{x_{n}} \\
& \frac{\partial\left(\varphi_{a}\right)_{i}}{\partial x_{n}}=\frac{-x_{i} g_{i}^{a}\left(x_{n}\right)}{x_{n}^{2}}+\frac{a x_{i} g_{i}^{a-1}\left(x_{n}\right)}{x_{n}} g_{i}^{\prime}\left(x_{n}\right) \text { and } \frac{\partial\left(\varphi_{a}\right)_{n}}{\partial x_{n}}=a x_{n}^{a-1}
\end{aligned}
$$

for any $i=1, \ldots, n-1$.

Hence $J\left(x, \varphi_{a}(x)\right)=a x_{n}^{a-n} G^{a}\left(x_{n}\right)$. By definition, the functions $g_{i}, i=1,2, \ldots$, $n-1$ are Lipschitz. So there exists a constant $M<+\infty$ such that

$$
g_{i}\left(x_{n}\right) \leq M x_{n} \quad \text { and } \quad g_{i}^{\prime}\left(x_{n}\right) \leq M
$$

for any $x_{n} \in[0,1]$ and $i=1,2, \ldots, n-1$. Using estimates for derivatives and the inequalities $x_{i} \leq x_{n}$ that are correct for all $x \in H_{1}$ we obtain the following estimate: $\left|D \varphi_{a}(x)\right| \leq c_{1} x_{n}^{a-1}$. In the same way we obtain also the two-sided estimate

$$
c_{2} x_{n}^{\alpha a} \leq\left|\varphi_{a}(x)\right|^{\alpha} \leq c_{3} x_{n}^{\alpha a} .
$$

Now we can check for which $q$ the homeomorphism $\varphi_{a}: H_{1} \rightarrow H_{g}$ is a $(p, q)$ quasiconformal homeomorphism. Let us start from the following estimate:

$$
\begin{gathered}
I_{a}=K_{p, q}\left(H_{1}\right)^{\frac{p q}{p-q}}=\int_{H_{1}}\left(\frac{\left|D \varphi_{a}(x)\right|^{p}}{\left|J\left(x, \varphi_{a}\right)\right|}\right)^{\frac{q}{p-q}} d x \\
\leq C \int_{0}^{1} \int_{0}^{x_{n}} \ldots \int_{0}^{x_{n}}\left(\frac{x_{n}^{p(a-1)}}{x_{n}^{a-n} G^{a}\left(x_{n}\right)}\right)^{\frac{q}{p-q}} d x_{1} \ldots d x_{n-1} d x_{n} \\
=C \int_{0}^{1} x_{n}^{(p(a-1)-a+n) \frac{q}{p-q}+n-1} G^{-a \frac{q}{p-q}}\left(x_{n}\right) d x_{n} .
\end{gathered}
$$

Hence, the quantity $I_{a}$ is finite if

$$
(p(a-1)-a+n) \frac{q}{p-q}+n-a(\gamma-1) \frac{q}{p-q}>0
$$

or

$$
q<n p /(a \gamma+p-a p) .
$$

Hence, the homeomorphism $\varphi_{a}$ is a $(p, q)$-quasiconformal homeomorphism.

Let us check the conditions of Theorem 5 . First we have to estimate the degree of integrability for the Jacobian $J_{a}$ of the homeomorphism $\varphi_{a}$ :

$$
\begin{aligned}
& J_{a}=\int_{H_{1}}(|J(x, \varphi)| w(\varphi(x)))^{\frac{r}{r-s}} d x \\
& \leq C \int_{0}^{1} \int_{0}^{x_{n}} \ldots \int_{0}^{x_{n}} x_{n}^{(a(\alpha+1)-n) \frac{r}{r-s}} G^{a \frac{r}{r-s}}\left(x_{n}\right) d x_{1} \ldots d x_{n-1} d x_{n} \\
& \leq C \int_{0}^{1} x_{n}^{(a(\alpha+1)-n) \frac{r}{r-s}+n-1+a \frac{r}{r-s}(\gamma-1)} d x_{n} .
\end{aligned}
$$


The integral $J_{a}$ converges if

$$
(a(\alpha+1)-n) \frac{r}{r-s}+n+a \frac{r}{r-s}(\gamma-1)>0,
$$

or

$$
s<\frac{a(\alpha+\gamma)}{n} r .
$$

Hence, the conditions of Theorem 5 are fulfilled if

$$
s<\frac{a(\alpha+\gamma)}{n} r, r<\frac{n q}{n-q} \text { and } q<\frac{n p}{a \gamma+p-a p} .
$$

Therefore

$$
s<\frac{a(\alpha+\gamma)}{n} \frac{n q}{n-q}<\frac{n p}{a(\gamma-p)} \frac{a(\alpha+\gamma)}{n}=\frac{p(\alpha+\gamma)}{\gamma-p}
$$

\section{Sobolev EMBedDings FOR SPACES With High DERIVATIVES}

This section is devoted to embedding theorems for weighted Sobolev spaces with high derivatives.

If for some $m$ and $p$ an embedding theorem for classical Sobolev spaces $W_{p}^{m}$ is correct, then, using a standard procedure, it is possible to obtain the corresponding embedding theorem for $m_{1} \geq m$ and $p_{1} \geq p$ also (see, for example, 9]). Here we adopt the scheme of $[9]$ to the case of weighted Sobolev spaces. The next lemma is the main technical result of this section.

Lemma 3. Let $D$ be a domain in $\mathbb{R}^{n}$. Suppose that for some $p_{0} \geq 1$ and $q_{0} \geq 1$ the embedding operator

$$
W_{p_{0}}^{1}(D, w) \hookrightarrow L_{q_{0}}(D, w)
$$

is bounded. Let $p \geq p_{0}$ and $\frac{1}{p}>\frac{1}{p_{0}}-\frac{1}{q_{0}}$. If $q$ is such that

then the embedding operator

$$
\frac{1}{p}-\frac{1}{q}=\frac{1}{p_{0}}-\frac{1}{q_{0}},
$$

$$
W_{p}^{1}(D, w) \hookrightarrow L_{q}(D, w)
$$

is bounded also.

Proof. Let a function $u$ belong to the space $W_{p}^{1}(D, w) \cap C_{0}^{\infty}(D)$. Using boundedness of the embedding operator

$$
W_{p_{0}}^{1}(D, w) \hookrightarrow L_{q_{0}}(D, w)
$$

we obtain the following estimate:

$$
\begin{gathered}
\left(\int_{D}|u|^{q} w(x) d x\right)^{\frac{1}{q_{0}}}=\left(\int_{D}\left(|u|^{\frac{q}{q_{0}}}\right)^{q_{0}} w(x) d x\right)^{\frac{1}{q_{0}}} \\
\leq C\left(\left(\int_{D}\left|\nabla\left(u^{\frac{q}{q_{0}}}\right)\right|^{p_{0}} w(x) d x\right)^{\frac{1}{p_{0}}}+\left(\int_{D}\left(|u|^{\frac{q}{q_{0}}}\right)^{p_{0}} w(x) d x\right)^{\frac{1}{p_{0}}}\right) \\
\leq C\left(\left(\int_{D}|u|^{p_{0} \frac{q-q_{0}}{q_{0}}}|\nabla u|^{p_{0}} w(x) d x\right)^{\frac{1}{p_{0}}}+\left(\int_{D}|u|^{p_{0} \frac{q-q_{0}}{q_{0}}}|u|^{p_{0}} w(x) d x\right)^{\frac{1}{p_{0}}}\right) .
\end{gathered}
$$


Applying the Hölder inequality we get

$$
\begin{aligned}
\left(\int_{D}|u|^{q} w(x) d x\right)^{\frac{1}{q_{0}}} & \leq C\left(( \int _ { D } | \nabla u | ^ { p } w ( x ) d x ) ^ { \frac { 1 } { p } } \cdot \left(\int_{D}|u|^{\left.p p_{0} \frac{q-q_{0}}{q_{0}\left(p-p_{0}\right)} w(x) d x\right)^{\frac{p-p_{0}}{p p_{0}}}}\right.\right. \\
& \left.+\left(\int_{D}|u|^{p} w(x) d x\right)^{\frac{1}{p}} \cdot\left(\int_{D}|u|^{p p_{0} \frac{q-q_{0}}{q_{0}\left(p-p_{0}\right)}} w(x) d x\right)^{\frac{p-p_{0}}{p p_{0}}}\right) .
\end{aligned}
$$

Because $q=p p_{0} \frac{q-q_{0}}{q_{0}\left(p-p_{0}\right)}$ we obtain finally

$$
\begin{aligned}
& \left(\int_{D}|u|^{q} w(x) d x\right)^{\frac{1}{q_{0}}} \\
& \quad \leq C\left(\left(\int_{D}|\nabla u|^{p} w(x) d x\right)^{\frac{1}{p}}+\left(\int_{D}|u|^{p} w(x) d x\right)^{\frac{1}{p}}\right) \cdot\left(\int_{D}|u|^{q} w(x) d x\right)^{\frac{p-p_{0}}{p p_{0}}} .
\end{aligned}
$$

Since $\frac{1}{q_{0}}-\frac{p-p_{0}}{p p_{0}}=\frac{1}{q}$ then

$$
\left\|u\left|L_{q}(D, w)\|\leq C\| u\right| W_{p}^{1}(D, w)\right\| .
$$

The lemma is proved.

Denote

$$
q_{m, D}^{*}(p)=\sup \left\{q \in \mathbb{R}^{+}: \text {the operator } W_{p}^{m}(D, w) \hookrightarrow L_{q}(D, w) \text { is bounded }\right\} .
$$

The following statements can be obtained directly from Lemma 3.

Corollary 3. If $D$ is a bounded domain in $\mathbb{R}^{n}, p \geq p_{0}$, then

$$
q_{1, D}^{*}(p) \geq \frac{p p_{0} q_{1, D}^{*}\left(p_{0}\right)}{p_{0} q_{1, D}^{*}\left(p_{0}\right)-p\left(q_{1, D}^{*}\left(p_{0}\right)-p_{0}\right)} .
$$

Corollary 4. If $D$ is a bounded domain in $\mathbb{R}^{n}, p \geq p_{0}$ and $m>1$, then

$$
q_{m, D}^{*}(p) \geq \frac{p p_{0} q_{1, D}^{*}\left(p_{0}\right)}{p_{0} q_{1, D}^{*}\left(p_{0}\right)-m p\left(q_{1, D}^{*}\left(p_{0}\right)-p_{0}\right)} .
$$

Proof. This corollary follows from the previous corollary by induction with respect to $m$.

Combining Theorem 5 and Corollary 4 we obtain finally

Theorem 9. Let domain $D \subset \mathbb{R}^{n}$ be an embedding domain and let there exist a w-weighted $(p, q)$-quasiconformal homeomorphism $\varphi: D \rightarrow D^{\prime}$ of the domain $D$ onto the bounded domain $D^{\prime}$.

If for some $s \leq r \leq n q /(n-q)$ the following inequality is correct,

$$
\int_{D}(|J(x, \varphi)| w(\varphi(x)))^{\frac{r}{r-s}}<+\infty,
$$

then an embedding operator

$$
i: W_{p}^{m}\left(D^{\prime}, w\right) \hookrightarrow L_{s^{*}}\left(D^{\prime}, w\right)
$$


is bounded if

$$
s^{*} \geq \frac{p s}{s-m(s-p)}
$$

\section{Solvability of Degenerate Elliptic EQUations}

In this section we apply an embedding theorem for Hilbert Sobolev spaces $W_{2}^{1}$ to a degenerate elliptic boundary problem.

Theorem 10. Let $D^{\prime} \subset \mathbb{R}^{n}$ be a quasi-isometrical image of an embedding domain $D$ and

$$
\int_{D^{\prime}} w(y)^{-\frac{n}{2}} d y<+\infty .
$$

Then an embedding operator

$$
i^{\prime}: W_{2}^{1}\left(D^{\prime}, w\right) \hookrightarrow L_{2}\left(D^{\prime}\right)
$$

is bounded.

Proof. By the conditions of the theorem there exists a $Q$-quasi-isometrical homeomorphism $\varphi: D \rightarrow D^{\prime}$ of the embedding domain $D$ onto $D^{\prime}$. For any function $u \in W_{2}^{1}\left(D^{\prime}, w\right)$ the composition $u \circ \varphi$ is weakly differentiable in the domain $D$, and the following estimates are correct for any $1 \leq q \leq 2$ :

$$
\begin{aligned}
\left\|u \circ \varphi \mid W_{q}^{1}(D)\right\| & =\left(\int_{D}|u \circ \varphi|^{q} d x\right)^{\frac{1}{q}}+\left(\int_{D}|\nabla(u \circ \varphi)|^{q} d x\right)^{\frac{1}{q}} \\
\leq & \left(\int_{D}|u \circ \varphi|^{q}\left(|J(x, \varphi)| w(\varphi(x))^{\frac{q}{2}} \frac{1}{\left(|J(x, \varphi)| w(\varphi(x))^{\frac{q}{2}}\right.} d x\right)^{\frac{1}{q}}\right. \\
& +\left(\int_{D}|\nabla u|^{q}\left|\bar{\varphi}^{\prime}(x)\right|^{q}\left(|J(x, \varphi)| w(\varphi(x))^{\frac{q}{2}} \frac{1}{\left(|J(x, \varphi)| w(\varphi(x))^{\frac{q}{2}}\right.} d x\right)^{\frac{1}{q}} .\right.
\end{aligned}
$$

By the Hölder inequality we have

$$
\begin{aligned}
& \left\|u \circ \varphi \mid W_{q}^{1}(D)\right\| \\
& \leq\left(\int_{D}\left(\frac{1}{|J(x, \varphi)| w(\varphi(x))}\right)^{\frac{q}{2-q}} d x\right)^{\frac{2-q}{2 q}}\left(\int_{D}|u|^{2}(\varphi(x)) w(\varphi(x))|J(x, \varphi)| d x\right)^{\frac{1}{2}} \\
& +\left(\int_{D}\left(\frac{\left|\bar{\varphi}^{\prime}(x)\right|^{2}}{|J(x, \varphi)| w(\varphi(x))}\right)^{\frac{q}{2-q}} d x\right)^{\frac{2-q}{2 q}}\left(\int_{D}|\nabla u|^{2}(\varphi(x)) w(\varphi(x))|J(x, \varphi)| d x\right)^{\frac{1}{2}} .
\end{aligned}
$$


Since $\varphi$ is the $Q$-quasi-isometrical homeomorphism, then by the change of variable formula in the Lebesgue integral, we obtain

$$
\begin{aligned}
&\left\|u \circ \varphi \mid W_{q}^{1}(D)\right\| \leq Q^{\frac{n}{q}}\left(\int_{D^{\prime}} w(y)^{\frac{q}{q-2}} d y\right)^{\frac{2-q}{2 q}}\left(\int_{D^{\prime}}|u|^{2} w(y) d y\right)^{\frac{1}{2}} \\
&+Q^{\frac{2-q+n}{q}}\left(\int_{D^{\prime}} w(y)^{\frac{q}{q-2}} d y\right)^{\frac{2-q}{2 q}}\left(\int_{D^{\prime}}|\nabla u|^{p} w(y) d y\right)^{\frac{1}{2}} \\
&=Q^{\frac{n}{q}} K(w)\left\|u\left|L_{2}\left(D^{\prime}, w\right)\left\|+Q^{\frac{2-q+n}{q}} K(w)\right\| \nabla u\right| L_{2}\left(D^{\prime} w\right)\right\| .
\end{aligned}
$$

By the previous inequality the composition operator

$$
\varphi^{*}: W_{2}^{1}\left(D^{\prime}, w\right) \rightarrow W_{q}^{1}(D), 1 \leq q \leq 2,
$$

is bounded.

Since $D$ is an embedding domain there exists the bounded embedding operator

$$
i: W_{q}^{1}(D) \hookrightarrow L_{\frac{n q}{n-q}}(D) .
$$

Now we choose a number $q$ such that $\frac{n q}{n-q}=2$ (i.e. $q=\frac{2 n}{n+2}$ ).

Since $\varphi$ is the $Q$-quasi-isometrical homeomorphism, the following composition operator acting on Lebesgue spaces,

$$
\left(\varphi^{-1}\right)^{*}: L_{2}(D) \rightarrow L_{2}\left(D^{\prime}\right),
$$

is bounded also [18.

Therefore the embedding operator $i^{\prime}: W_{2}^{1}\left(D^{\prime}, w\right) \hookrightarrow L_{2}\left(D^{\prime}\right)$ is bounded as a composition of bounded operators $\varphi^{*},\left(\varphi^{-1}\right)^{*}$ and the bounded embedding operator $i$.

Define an inner product in the weighted space $W_{2}^{1}(D, w)$ as:

$$
\langle u, v\rangle=\int_{D} \nabla u \cdot \nabla v w(x) d x,
$$

for any $u, v \in W_{2}^{1}(D, w)$.

Consider the Dirichlet problem for the degenerate elliptic equation:

$$
\begin{gathered}
\operatorname{div}(w(x) \nabla u)=f, \\
\left.u\right|_{\partial D}=0
\end{gathered}
$$

in a bounded domain $D$ for the weight $w \in C^{1}(D)$.

Theorem 11. Let $f \in L_{2}(D)$ and $\int_{D} w(y)^{-\frac{n}{2}} d x<+\infty$. Then there exists a unique weak solution $u \in \stackrel{\circ}{W}_{2}^{1}(D, w)$ of the problem $(1),(2)$.

Proof. The function $f \in L_{2}(D)$ induces a linear functional $F: L_{2}(D) \rightarrow R$ by the standard rule

$$
F(\phi)=\int_{D} f(x) \phi(x) d x .
$$

By Theorem 10 there exists a bounded embedding operator $i: \stackrel{\circ}{W}_{2}^{1}(D, w) \hookrightarrow L_{2}(D)$. 
Therefore

$$
\begin{aligned}
|F(\phi)| \leq \| f \cdot \phi \mid & L_{1}(D) \| \\
& \leq\left\|f\left|L_{2}(D)\|\cdot\| \phi\right| L_{2}(D)\right\| \leq C\left\|f\left|L_{2}(D)\|\cdot\| \phi\right| \stackrel{\circ}{\stackrel{1}{W}_{2}}(D, w)\right\| .
\end{aligned}
$$

Hence, $F$ is a bounded linear functional in the Hilbert space $\stackrel{\circ}{W_{2}}(D, w)$. By the

Riesz representation theorem [20] there exists a unique function $u \in \stackrel{\circ}{W^{1}}{ }_{2}(D, w)$ such that

or

$$
F(\phi)=\langle u, \phi\rangle=\int_{D} \nabla u \cdot \nabla \phi w(x) d x
$$

$$
\int_{D} \nabla u \cdot \nabla \phi w(x) d x=\int_{D} f(x) \phi(x) d x .
$$

Therefore $u$ is the unique weak solution of the problem (1), (2).

\section{REFERENCES}

[1] Kufner A. Weighted Sobolev spaces, Leipzig, Teubner-Texte zur Mathematik, 1980. MR664599(84e:46029)

[2] Turesson B. O. Nonlinear Potential Theory and Weighted Sobolev Spaces, Berlin, Springer (Lecture Notes in Mathematics, no. 1736), 2000. MR1774162 (2002f:31027)

[3] Gurka P., Opic B. Continuous and compact embeddings of weighted Sobolev spaces. I, Czech. Math. J., 1988, vol. 38, 113, pp. 730-744. MR962916 (89j:46034)

[4] Gurka P., Opic B. Continuous and compact embeddings of weighted Sobolev spaces. II., Czech. Math. J., 1989, vol. 39, 114, pp. 78-94. MR983485 (90e:46027)

[5] Gurka P., Opic B. Continuous and compact embeddings of weighted Sobolev spaces. III, Czech. Math. J., 1991, vol. 41, 116. pp. 317-341. MR1105449 (93a:46056)

[6] Antoci F. Some necessary and some sufficient conditions for the compactness of the embedding of weighted Sobolev spaces, Ricerche Mat., 2003, vol. 52, 1, pp. 55-71. MR2091081 (2005f:46062)

[7] Besov O. V. The Sobolev embedding theorem for a domain with an irregular boundary, Doklady Mathematics, 2000, vol. 62, 1, pp. 22-25. MR1788313 (2001g:46067)

[8] David G., Semmes S. Strong $A_{\infty}$-weights, Sobolev inequalities and quasiconformal mappings. Lecture Notes in Pure and Appl. Math., 1990, vol. 122, pp. 101-111. MR.1044784 (91c:30037)

[9] Gol'dshtein V., Gurov L. Applications of change of variables operators for exact embedding theorems, Integral Equations Operator Theory, 1994, vol. 19, 1, pp. 1-24. MR1271237 (95c:46045)

[10] Gol'dshtein V., Ramm A. G. Compactness of the embedding operators for rough domains, Math. Inequalities and Applications, 2001, vol. 4, 1, pp. 127-141. MR.1809846 (2001m:46075)

[11] Vodop'yanov S. K., Ukhlov A. Set functions and their applications in the theory of Lebesgue and Sobolev spaces. II, Siberian Adv. Math., 2005, vol. 15, pp. 91-125. MR2141791

[12] Burenkov V. I. Sobolev Spaces on Domains, Stuttgart: Teubner-Texte zur Mathematik, 1998. MR.1622690 (99g:46040)

[13] Stein E. Harmonic analysis: real-variable methods, orthogonality, and oscillatory integrals, Princeton: Princeton Univ. Press, 1993. MR1232192 (95c:42002)

[14] Kilpelainen T. Weighted Sobolev spaces and capacity, Ann. Acad. Sci. Fenn. Ser. A. I. Math., 1994, vol. 19, pp. 95-113. MR.1246890 (95h:46054)

[15] Heinonen J., Kilpelainen T., Martio O. Nonlinear potential theory of degenerate elliptic equations, Oxford, Oxford Univ. Press, 1993. MR1207810 (94e:31003)

[16] Maz'ya V. Sobolev spaces, Berlin: Springer-Verlag, 1985. MR817985 (87g:46056)

[17] Gol'dshtein V. M., Reshetnyak Yu. G. Quasiconformal mappings and Sobolev spaces, Dordrecht, Boston, London: Kluwer Academic Publishers, 1990. MR.1136035 (92h:46040)

[18] Vodop'yanov S. K., Ukhlov A. Set functions and their applications in the theory of Lebesgue and Sobolev spaces. I, Siberian Adv. Math., 2004, vol. 14, 4, pp. 78-125. MR2125997 
[19] Vodop'yanov S. K., Ukhlov A. Mappings associated with weighted Sobolev spaces, Contemporary Mathematics, 2007, vol. 424 (to appear)

[20] Riesz F. Sur les opérations fonctionelles linéaires, C. R. Acad. Sci. Paris, 1909, vol. 149, pp. 974-977.

[21] Smith W., Stegenga D. A. Hölder domains and Poincaré domains, Trans. Amer. Math. Soc., 1990, vol. 319, pp. 67-100. MR978378 (90i:30012)

Department of Mathematics, Ben Gurion University of the Negev, P.O. Box 653, 84105 Beer Sheva, IsRael

Department of Mathematics, Ben Gurien Unniversity of the Negev, P.O. Box 653, 84105 Beer Sheva, Israel 